Oswaldo de Almeida Pastore ${ }^{1}$

Wilson Siguemasa Iramina²

\section{A influência da temperatura na eficiência de filtros com carga eletrostática usados na proteção respiratória*}

\author{
The influence of temperature over the efficiency of electrostatic
} charged filters for respiratory protection

\footnotetext{
1 Tecnólogo Mecânico, mestre pelo Departamento de Engenharia de Minas e de Petróleo da Escola Politécnica da Universidade de São Paulo.

${ }^{2}$ Engenheiro de Minas e de Segurança do Trabalho, Professor da Escola Politécnica da Universidade de São Paulo.

\begin{abstract}
*Artigo baseado na dissertação de mestrado de Oswaldo de Almeida Pastore intitulada A influência da temperatura na eficiência de filtros mecânicos com carga eletrostática usados em respiradores em ambiente de mineração, apresentada em 2009 ao Departamento de Engenharia de Minas e de Petróleo da Escola Politécnica da Universidade de São Paulo.
\end{abstract}

Contato:

Oswaldo de Almeida Pastore

Avenida Roberto Gordon, 138

Diadema - SP - 09990-901

E-mail:

pastore1966@gmail.com

\section{Resumo}

O uso de filtros e respiradores contra particulados é extremamente comum na indústria em geral. As normas utilizadas para a avaliação desses equipamentos definem critérios de testes e aprovação que visam garantir que os filtros continuem eficientes ao longo do tempo. Fatores como tipo, tamanho, velocidade, carga da partícula e temperatura são, em geral, considerados nos testes de aprovação. O objetivo deste artigo foi verificar o comportamento de filtros para particulados tipo eletreto usados em respiradores, expostos às condições térmicas semelhantes às encontradas em ambiente de trabalho. Simularamse, em laboratório, a temperatura e o tempo de exposição a que os mesmos podem estar sujeitos, medindo e comparando os resultados obtidos no sentido de atenderem as recomendações normativas, tanto nacionais como internacionais. Filtros e peças faciais de diferentes fabricantes foram expostos a diferentes temperaturas, sendo mantidos nestas por, no mínimo, 24 horas. Testes de penetração utilizando vazão de 85 l/min e partículas de $\mathrm{NaCl}$, da ordem de 0,3 a $0,6 \mu \mathrm{m}$, que são consideradas as Partículas de Tamanhos Mais Penetrantes (MPPS), foram realizados antes e depois de cada período de exposição. A menor e a maior variações encontradas foram de $2,53 \%$ até $18 \%$, respectivamente.

Palavras-chave: filtro mecânico, partícula, eletrostático, segurança, equipamento de proteção individual.

\section{Abstract}

Particle filters are very commonly used in industries in general. The standards adopted for the approval of these filters define tests and approval criteria aiming at guaranteeing the efficiency of the filters in the long run. Facts such as: type, size, speed, particle charge and temperature are considered on the approval tests. This article aims at checking the way eletrect particulated filters for respirators act when exposed to similar thermal conditions as the ones found in work environment. This was obtained by simulating in lab the temperature and time of exposure the respirators could be subjected to and by measuring and comparing the results in order to comply with both national and international standards recommendations. Filters and face pieces from different manufactures were tested in different temperatures and maintained under these conditions for at least 24-hours. Penetration tests were held before and after each period of exposure using $85 \mathrm{l} / \mathrm{min}$ flow rate and 0.3 to $0.6 \mu \mathrm{m} \mathrm{NaCl}$ particles, which are considered the Most Penetranting Particle Size (MPPS). The smallest and largest variations found were $2.53 \%$ to $18 \%$, respectively.

Keywords: mechanical filter, particle, electrostatic, safety, personal protective equipment. 


\section{Introdução}

Para efeito da pesquisa apresentada nesta publicação, foram utilizados, durante o estudo, Equipamentos de Proteção Individual (EPI) específicos para a proteção respiratória e utilizados, em particular, na proteção contra particulados em suspensão. Estes tipos de EPI são comumente chamados de máscaras, sendo técnica e genericamente definidos como Equipamentos de Proteção Respiratória (EPR) ou, apenas, respiradores.

Um respirador pode ser fabricado em material elastomérico, borracha ou outro material que permita a acomodação deste sobre a face, cobrindo as vias respiratórias superiores. Este tipo de respirador utiliza filtros intercambiáveis acoplados à peça facial; um respirador também pode ser do tipo peça semifacial filtrante (PFF), podendo este ser constituído parcial ou totalmente de material filtrante. Em geral, os filtros são fabricados com mantas filtrantes sobrepostas por camadas de mantas de Tecidos Não Tecidos (TNT), as quais recebem uma configuração geométrica tal que permita o uso como um respirador.

Garantir que os EPR atendam aos objetivos pretendidos é de suma importância para se preservar a saúde do trabalhador. As especificações técnicas para a aprovação dos EPR estão definidas em normas técnicas nacionais e internacionais específicas para cada tipo de respirador. Estas normas, no caso de filtros contra partículas, definem basicamente o tipo de partícula ou névoa a ser utilizado, tamanho, velocidade, condicionamento, limites de resistências à inalação e penetração/eficiência do filtro.

A penetração/eficiência é medida pela capacidade do filtro reter uma dada porcentagem da quantidade de partícula à qual o filtro foi exposto. É por meio destes testes que se pode determinar se um filtro irá ou não oferecer a proteção adequada ao trabalhador.

Na prática, o usuário do filtro terá somente dois parâmetros para saber se o seu filtro está realmente filtrando ou não os contaminantes, são eles: o aumento da resistência à respiração e/ou o odor. No caso dos particulados em específico, a questão do odor é raramente percebida. Deste modo, o trabalhador pode despercebidamente estar utilizando um filtro que não irá ajudar a proteger a sua saúde. Daí a importância das empresas terem um programa adequado de substituição programada dos filtros, sempre zelando pela qualidade dos produtos utilizados, sem falar nas questões de ajuste à face e uso adequado do equipamento.

No caso de filtros contra particulados, fibras de diferentes materiais podem ser empregadas na fabricação dos mesmos, como lã de carneiro, poliéster, poliuretano, polipropileno, entre outras. Estas fibras, quando entrelaçadas ou depositadas de modo aleatório umas sobre as outras, formam uma manta que pode ser utilizada como filtro. Estas mesmas fibras, quando carregadas eletrostaticamente, aumentam de forma significativa a eficiência da filtração. Os filtros carregados eletrostaticamente são chamados de filtros eletretos ou elétricos. Nos casos em que a carga eletrostática exerce um papel preponderante nos mecanismos de captura das partículas, a perda ou a diminuição desta pode levar um filtro, outrora eficiente, à condição de inapropriado para a proteção do trabalhador.

Diversos autores (MOYER; BERGMAN, 2000; MOTYL; Lowkis, 2006; FJELD; OWENS, 1988; BROWN, 2001) descreveram que o decaimento da carga eletrostática pode ocorrer por motivos como, por exemplo, exposição à alta umidade, radiação ionizante, aerossóis carregados eletricamente, partículas oleosas, alta temperatura, exposições intermitentes com cloreto de sódio $(\mathrm{NaCl})$ ou em função do tempo.

Sabendo-se, então, que os fatores temperatura elevada e umidade são comuns em ambientes de trabalho, fica a preocupação a respeito de quanto estas condições poderão afetar a eficiência dos filtros para particulados tipos eletreto quando utilizados em ambientes com alta temperatura.

Brown (1989), em sua pesquisa, constata que filtros mecânicos carregados eletricamente, quando submetidos a variações da temperatura acima de $90^{\circ} \mathrm{C}$ e até $110^{\circ} \mathrm{C}$, tinham, como consequência, um aumento da penetração, com uma estabilização da eficiência ocorrendo após períodos de aproximadamente 10 a 30 horas de exposição.

Martin (2000), em seus estudos baseados na certificação americana, conforme a norma 42 CFR 84 (ESTADOS UNIDOS, 1995), que classifica os filtros mecânicos em função do tipo de aerossol ao qual o filtro será exposto, constatou que, apesar de ser muito improvável que ocorra a degradação total da carga eletrostática do filtro exposto ao ambiente de trabalho, não se pode descartar a possibilidade de um certo grau de degradação ocorrer em função do uso diário, de armazenamento em condições adversas ou, mais importante ainda, da exposição a aerossóis industriais. Finalmente conclui que estudos adicionais necessitam ser realizados nesta área para avaliar essas possibilidades.

Os estudos de Motyl e Lowkis (2006) demonstram os resultados de testes comparativos entre amostras de mídias filtrantes tipo P1 e P2. Onde as amostras foram submetidas a diversas condições de umidade, medindo o decaimento da carga das amostras, as mantas P2 apresentaram uma vida útil maior, em termos de manutenção das cargas eletrostáticas, em relação à manta P1.

Segundo Cheng, Holmes e Fan (2006), a penetração de fibras de asbesto em filtros mecânicos armazenados durante uma semana à temperatura de $38^{\circ} \mathrm{C}$ e umidade de $90 \%$ aumentou de 0 a $3 \%$ para $20 \%$. Os únicos filtros que mantiveram a performance constante, independentemente das condições de teste, foram os filtros de alta 
eficiência High Efficiency Particulate Air (HEPA). Alguns filtros constituídos de material de baixa condutividade podem conter cargas eletrostáticas. Estas cargas podem ser perdidas sob condições de alta temperatura e alta umidade.

A utilização de filtros contra particulados, fabricados com fibras poliméricas carregadas eletrostaticamente, que possuem alta eficiência, baixa resistência, baixo custo e maior conforto, é cada vez mais frequente e popular, consequentemente, seu mercado está em expansão.

Normas nacionais e internacionais indicam as condições de pré-condicionamento dos filtros antes de os aprovarem para uso comercial, visando, assim, evitar a degradação dos mesmos. Entretanto, essa medida por si só não previne ou alerta sobre qualquer problema de degradação das características de filtração que possa vir a ocorrer no futuro.

Muitas vezes, o único meio de proteção do trabalhador é o uso do respirador, portanto, se o filtro, por uma razão ou outra, deixar de proporcionar a proteção adequada, o trabalhador terá uma falsa impressão de segurança, o que, consequentemente, poderá resultar em sérios danos à sua saúde. É importante observar que os meios que o usuário dispõe para avaliar a eficiência do respirador que utiliza são o aumento da resistência à respiração e/ou o odor, percebidos por ele próprio.

Apesar de pouco provável que as condições desfavoráveis para a perda da eficiência de filtração ocorram simultaneamente e sistematicamente no ambiente de trabalho, não se pode descartar a possibilidade de que, durante a jornada de trabalho, os filtros fiquem expostos por longos períodos à elevada temperatura.

Não existem recomendações, seja por exigência de norma ou mesmo por iniciativa dos próprios fabricantes, quanto aos cuidados que devem ser observados no armazenamento e em relação à exposição dos EPR à temperatura elevada, nem quanto aos seus efeitos potenciais em filtros tipo eletretos.

Finalmente, considerando que a degradação da carga eletrostática afeta diretamente a eficiência do filtro e, consequentemente, pode acarretar em dano à saúde do trabalhador, o estudo realizado pode ter um caráter relevante para futuras revisões de normas e orientações para os EPR atuais e, também, em desenvolvimentos de novos materiais filtrantes.

Assim, o objetivo deste trabalho foi verificar, por meio de testes em laboratório, o comportamento dos filtros tipo eletretos quanto à penetração de $\mathrm{NaCl}$ mediante permanência em estufa com temperaturas similares às encontradas no ambiente de trabalho, variando o tempo de permanência nas diversas temperaturas. Foi também avaliado se os resultados de penetração obtidos com o aumento da temperatura enquadram-se nas exigências normativas brasilei- ras e estrangeiras, fazendo, ainda, uma comparação dos resultados obtidos entre diferentes fabricantes e classes de filtros.

\section{Metodologia}

A metodologia empregada foi a análise de resultados de testes em laboratório e a busca de informações junto a um fabricante de EPI e fabricantes de matériaprima utilizada na produção de filtros tipo eletretos.

Neste estudo, foram utilizados Peças Faciais Filtrantes (PFF) e filtros fabricados a partir de mantas planas carregadas eletrostaticamente. As gramaturas dos materiais filtrantes variaram entre 13 e $83 \mathrm{~g} / \mathrm{m}$.

O laboratório e os equipamentos utilizados foram cedidos pela empresa MSA do Brasil Equipamentos e Instrumentos de Segurança Ltda. e a coleta de dados deu-se por meio da realização de testes de penetração inicial com $\mathrm{NaCl}$ em filtros mecânicos. Foram usados os parâmetros normativos de aprovação com relação à penetração inicial e ao pré-condicionamento de filtros para particulados, como definidos nas normas EN 143 (COMISSÃO EUROPÉIA DE NORMALIZAÇÃO, 2000), EN 149 (COMISSÃO EUROPÉIA DE NORMALIZAÇÃO, 2001), NBR 13697 (ASSOCIAÇÃO BRASILEIRA DE NORMAS TÉCNICAS, 1996a), NBR 13698 (ASSOCIAÇÃO BRASILEIRA DE NORMAS TÉCNICAS, 1996b) e 42 CFR 84 (ESTADOS UNIDOS, 1995).

As amostras utilizadas neste estudo foram adquiridas no mercado varejista brasileiro da região da grande São Paulo, de 5 fabricantes distintos; estas amostras foram divididas em 8 grupos em função do tipo, classe e fabricante. O número de fabricantes, classificação dos filtros, tamanho dos grupos e quantidade de amostras estão detalhados no Quadro 1.

Os grupos de amostra identificados como 5 a 8,11 a 14 , do fabricante C, e 15,16 e 22 a 26 , do fabricante $\mathrm{D}$, apesar de serem produtos do mesmo fabricante, são de lotes distintos.

Apesar dos produtos utilizados nesta pesquisa possuírem aprovação brasileira, atendendo às normas NBR 13697 (ASSOCIAÇÃO BRASILEIRA DE NORMAS TÉCNICAS, 1996a) ou NBR 13698 (ASSOCIAÇÃO BRASILEIRA DE NORMAS TÉCNICAS, 1996b), todos, independentemente de fazerem referência à aprovação americana, foram testados conforme a norma 42 CFR Part 84 (ESTADOS UNIDOS, 1995); ou seja, vazão de teste de 85 l/min., concentração de $2 \%$ de sal e neutralizador de cargas das partículas ligado.

\section{Instrumentação}

Por questões de praticidade, precisão e velocidade de operação para a realização dos testes de penetração em filtros para particulados, aliadas ao objetivo de medir a variação dos resultados, dentre os equipamentos 
Quadro 1 Quantidades e tipos de filtros utilizados

\begin{tabular}{|c|c|c|c|c|}
\hline $\begin{array}{c}\text { Fabricante/Grupo de } \\
\text { Amostras }\end{array}$ & Qtd. & Tipo & Classe & Norma \\
\hline A-1 e 2 & 2 & Dobrável & P1 & EN/ NBR \\
B-3 e 4 & 2 & Dobrável & P1 & EN / NBR \\
C-5 a 8 & 4 & Plano & P2 / N95 & NBR/42 CFR \\
D-9 e 10 & 2 & Plano & P1 & EN / NBR \\
C-11 a 14 & 4 & Plano & P2/ N95 & NBR/42 CFR \\
D-15 e 17 & 3 & Moldado & PFF1 & EN / NBR \\
E-18 a 22 & 5 & Moldado & PFF2 & EN / NBR \\
D-23 a 26 & 4 & Moldado & P2/ N95 & NBR/42 CFR \\
\hline
\end{tabular}

Notas:

Tipo: refere-se à forma geométrica do filtro ou respirador.

Classe: é o modo como as respectivas normas classificam os filtros ou respiradores em função da sua eficiência de filtração.

Norma: indica a norma de aprovação nacional e/ou equivalente internacional.

disponíveis, Moore's e TSI, do laboratório da empresa MSA do Brasil, optou-se pelo uso do equipamento TSI modelo 8130. Vale destacar que este modelo foi homologado pelo National Institute for Occupational Safety and Health (NIOSH) para aprovação dos filtros para particulados, conforme a norma 42 CFR 84 (ESTADOS UNIDOS, 1995). No entanto, o mesmo não está descrito nas normas brasileiras NBR 13697 (ASSOCIAÇÃO BRASILEIRA DE NORMAS TÉCNICAS, 1996a) e NBR 13698 (ASSOCIAÇÃO BRASILEIRA DE NORMAS TÉCNICAS, 1996b), não podendo ser usado no Brasil para fins de aprovação de produtos pelos laboratórios competentes ${ }^{3}$.

Em seu estudo comparativo entre os resultados de penetração obtidos pelos equipamentos Moore's e TSI, Camargo (2007) concluiu que existe uma relação de praticamente 1:1 em todos os níveis de penetração, possibilitando assim a viabilidade técnica do uso do equipamento da TSI para verificação da adequação dos produtos aprovados conforme resultados obtidos no equipamento Moore's.

O atomizador usado em conjunto com o equipamento TSI 8130 foi o modelo 8110 A, específico para solução de NaCl, também do fabricante TSI Incorporated. Este atomizador pode gerar aerossol em uma faixa de $1,0 \mu \mathrm{g} / \mathrm{m}^{3}$ a $200 \mathrm{mg} / \mathrm{m}^{3}$. O atomizador $8118 \mathrm{~A}$ usa um impactador que produz uma faixa estreita de distribuição do aerossol, o qual possui uma distribuição geométrica padrão ( $\sigma \mathrm{g})$ menor que 1,86 (TSI INCORPORATED, 2004). O aerossol de NaCl possui um tamanho médio mássico aerodinâmico típico de
0,26 $\mu \mathrm{m}$, um diâmetro médio típico de 0,08 $\mu \mathrm{m}$ e uma concentração de $15 \mathrm{mg} / \mathrm{m}^{3}$ a $20 \mathrm{mg} / \mathrm{m}^{3}$ em diluição a 70 litros por minuto $(1 / \mathrm{m})$.

\section{Cálculo da penetração}

O desempenho de um filtro para particulados é medido em função da sua capacidade de permitir que partículas sólidas ou oleosas, de tamanho e concentração conhecidos, passem por ele ou que nele fiquem retidas.

A medição da eficiência do filtro é expressa em termos porcentuais e indica a quantidade da massa das partículas que este retém ou deixa passar. Portanto, é comum, além do termo penetração (\% que transpassou o filtro), usar o termo eficiência (\% que o filtro reteu). Por exemplo, se for obtida a leitura no equipamento de teste de filtros no valor de $20 \%$, pode-se dizer que o filtro teve uma penetração de $20 \%$ ou que sua eficiência é de 80\%. As normas brasileiras NBR 13697 (ASSOCIAÇÃO BRASILEIRA DE NORMAS TÉCNICAS, 1996a) e NBR 13698 (ASSOCIAÇÃO BRASILEIRA DE NORMAS TÉCNICAS, 1996b) e da União Européia, EN 143 (COMISSÃO EUROPÉIA DE NORMALIZAÇÃO, 2000) e EN 149 (COMISSÃO EUROPÉIA DE NORMALIZAÇÃO, 2001), utilizam o termo penetração, enquanto a norma norte-americana 42 CFR 84 (ESTADOS UNIDOS, 1995) usa o termo eficiência.

Independente da nomenclatura utilizada, a penetração ou eficiência consiste na seguinte relação:

\footnotetext{
${ }^{3}$ Conforme conversas pessoais com fabricantes de elementos filtrantes e de EPI, o equipamento TSI 8130 é usado universalmente, sendo, no caso do Brasil, usado para fins de pesquisas e controle da qualidade.
} 
$P=(C J / C m) \times 100 \quad$ ou $\quad E=C m-(C j / C m) \times 100$

Onde:

$\mathrm{P}=$ Penetração $(\%)$

$\mathrm{E}=$ Eficiência (\%)

$\mathrm{Cm}=$ Concentração a montante

$\mathrm{Cj}=$ Concentração a jusante

A relação entre eficiência e penetração é a seguinte:

$\mathrm{E}=100-\mathrm{P}$

\section{Condições de condicionamento térmico}

Os filtros foram acondicionados em uma estufa onde a temperatura foi ajustada conforme o controle disponível na mesma. O acompanhamento da temperatura foi realizado por meio do uso de um termômetro de bulbo, com faixa de medição de 0 a $200^{\circ} \mathrm{C}$.

\section{Calibração dos equipamentos}

Todos os equipamentos utilizados neste experimento foram adequadamente calibrados contra padrões rastreáveis da Rede Brasileira de Calibração (RBC) e/ou padrões internacionais e, ainda, conforme padrões definidos pela empresa TSI Incorporated.

\section{Calibração do CERTITEST® Modelo 8130}

Os equipamentos de testes de filtros medem basicamente a penetração do aerossol e a resistência à respiração ou à perda de carga. As unidades destas medições são respectivamente porcentagem (\%) e milímetros de coluna d'água $\left(\mathrm{mmH}_{2} \mathrm{O}\right)$. Outra unidade extremamente importante e que afeta diretamente os resultados da medição é a vazão, que é medida em litros por minuto (1/min).

\section{Procedimento de calibração do equipamento de teste}

A calibração foi feita diariamente seguindo a metodologia e os padrões estabelecidos pelo fabricante, conforme descrito no manual do equipamento TSI 8130 (TSI INCORPORATED, 2008). A calibração, conforme estabelecida, é, na verdade, uma validação dos parâmetros-chave do sistema de medição, como segue:

\section{- Verificação da vazão e perda de carga}

A verificação da exatidão dos resultados da vazão e da perda de carga é feita por intermédio do uso de uma placa de orifícios, que possui uma resistência conhecida para uma dada vazão.

A placa é posicionada no equipamento como se fosse realizar um teste de filtro, porém, a vazão é ajustada conforme indicado na placa, neste caso, 32 l/min. A leitura da perda de carga deve ser a mesma que foi indicada na placa e é obtida por leitura direta no painel do equipamento.

\section{- Verificação da penetração}

O procedimento de validação foi projetado para testar vários aspectos do equipamento de teste em filtros. Um deles é verificar o tamanho das partículas geradas por meio da comparação dos resultados da medição da penetração em filtros, em que o nível de penetração do aerossol é conhecido. Adicionalmente, esta técnica provê a medição dos seguintes parâmetros: tamanho da distribuição do aerossol, medição da vazão, perda de carga, operação adequada dos fotômetros e operação geral do sistema.

Para tal validação (calibração), foram utilizadas folhas de filtros com uma faixa de penetração conhecida. Estes filtros são do tipo fibra de vidro e são fornecidos pela própria TSI, no formato de discos com 125 mm de diâmetro.

Os discos são acompanhados de cinco gráficos, os quais indicam a faixa de penetração esperada para cada combinação da quantidade de sobreposição dos discos. Desta forma, por exemplo, quando se submete um disco ao teste de penetração a uma vazão de 85 l/min e é obtido um resultado de resistência de $25 \mathrm{mmH}_{2} \mathrm{O}$, a penetração deve ser de aproximadamente $11,6 \%$, podendo estar, no máximo, na faixa de $8,8 \%$ a $14,6 \%$. Este procedimento deve se repetir sobrepondo-se os discos, obtendose, desta forma, uma verificação em toda a faixa de medição do equipamento ou, pelo menos, na faixa mais utilizada para testes. Os gráficos cobrem uma faixa de $22 \mathrm{mmH}_{2} \mathrm{O}$ de resistência e penetração de $14,4 \%$ a uma resistência de $140 \mathrm{mmH}_{2} \mathrm{O}$ e penetração de $0,08 \%$.

\section{- Parâmetros de maior influência na repetibilidade da medição da penetração}

Basicamente, o resultado da penetração em um filtro é resultante de três parâmetros: o tamanho do aerossol, o tamanho da distribuição do aerossol e a vazão.

O relacionamento dos parâmetros "tamanho da partícula”, "vazão" e "penetração" em um filtro é apresentado no manual de operações do equipamento de testes TSI 8130, o qual indica que partículas de diâmetro de $0,1 \mu \mathrm{m}$ apresentam penetração máxima de 15\% para vazão de 32 1/min e de 18\% para vazão de 54 l/min. Diâmetros de partículas maiores ou menores que $0,1 \mu \mathrm{m}$ apresentam penetrações menores, por exemplo, partículas de diâmetro de 0,02 $\mu \mathrm{m}$ apresentam uma penetração de $2 \%$ para uma vazão de $32 \mathrm{l} / \mathrm{min}$ e $3 \%$ para vazão de $54 \mathrm{l} / \mathrm{min}$. Por sua vez, as partículas de diâmetro de $0,4 \mu \mathrm{m}$ apresentam penetração de $0,8 \%$ e $1,1 \%$ para vazões respectivamente de 32 $\mathrm{l} / \mathrm{min}$ e $54 \mathrm{l} / \mathrm{min}$. Desta forma, observa-se que a variação da penetração em função da vazão/velocidade das partículas é bem menos expres- 
siva que a variação do diâmetro da partícula. O tamanho da distribuição do aerossol também exerce um efeito significativo na penetração, pois a mesma diminui com o aumento no tamanho da distribuição geométrica. Esta é, geralmente, medida e referenciada como desvio geométrico padrão (GSD). O aerossol polidisperso utilizado nos testes de penetração possui um GSD maior que 1,2 .

Partículas com diâmetro de 0,2 $\mu \mathrm{m}$ apresentam uma penetração de aproximadamente $15 \%$ para um GSD de 1,2 e penetração de $0,5 \mu \mathrm{m}$ para GSD de 2,0 (TSI INCORPORATED, 2004).

\section{Classificação dos filtros mecânicos em função da eficiência}

As normas nacionais e internacionais classificam os filtros mecânicos em função da sua capacidade de reter particulados de tamanho conhecido.

A norma 42 CFR Part 84 (ESTADOS UNIDOS, 1995), publicada pelo NIOSH, classifica os filtros da seguinte forma: séries N, R e P com três níveis de eficiência para cada classe, 95\%, 99\% e 99,97\%, todos testados com partículas consideradas MPPS, com o diâmetro de 0,3 $\mu \mathrm{m}$ (Diâmetro Aerodinâmico Médio Mássico - MMAD). As séries $\mathrm{N}$ são testadas contra leve degradação por $\mathrm{NaCl}$ e as séries $\mathrm{R}$ e $\mathrm{P}$, contra severa degradação de Di Octil Ftalato (DOP).

A NBR 12543 (ASSOCIAÇÃO BRASILEIRA DE NORMAS TÉCNICAS, 1999) define diâmetro aerodinâmico de uma partícula como diâmetro de uma partícula esférica, com densidade unitária, que possui a mesma velocidade terminal que a partícula considerada. Esta mesma norma define MMAD como o ponto na distribuição de tamanho das partículas em que a metade da massa das partículas tem diâmetro menor que o diâmetro aerodinâmico médio mássico e a outra metade tem diâmetro maior.

Já as normas NBR 13697 (ASSOCIAÇÃO BRASILEIRA DE NORMAS TÉCNICAS, 1996a) e EN 143 (COMISSÃO EUROPÉIA DE NORMALIZAÇÃO, 2000) classificam os filtros mecânicos da seguinte forma: classes P1, P2 e P3, sendo a máxima penetração inicial de $\mathrm{NaCl}$ permitida para cada classe, respectivamente, de $20 \%$, $6 \%$ e $0,05 \%$, com tamanho de partículas de $0,06 \mu \mathrm{m}$.

As normas NBR 13698 (ASSOCIAÇÃO BRASILEIRA DE NORMAS TÉCNICAS, 1996b) e EN 149 (COMISSÃO EUROPÉIA DE NORMALIZAÇÃO, 2001), específicas para PFF, classificam os respiradores como segue: classes PFF1, PFF2 e PFF3, sendo a máxima penetração inicial de $\mathrm{NaCl}$ para cada classe, respectivamente, de $20 \%, 6 \%$ e $3 \%$, exceto para a norma européia, segundo a qual a penetração para o PFF3 é de 1\%. As normas brasileiras aqui indicadas definem o ensaio com óleo de parafina para os filtros classes P2, P3, PFF-2 e PFF-3; as normas européias, por sua vez, definem-no para todas as classes.

\section{Origem do filtro eletreto e princípios de funciona- mento}

A partir do entendimento dos mecanismos de captura das partículas pelas fibras, devido à carga elétrica de uma ou ambas, começou-se a produzir filtros nos quais as fibras eram carregadas eletrostaticamente. Em 1931, surgiu o filtro de lã carregado com resina, que foi chamado de filtro Hansen. Para produzir esse filtro, é necessário que ocorra, primeiramente, a impregnação da superfície das fibras da lã com pequenas partículas de resina. Então, por meio de ações mecânicas subsequentes, como cardamento e posterior calandragem da manta de lã, as partículas de resina adquirem uma carga negativa e a lã, uma carga positiva. Estes filtros fazem uso de fibras de lã com aproximadamente $20 \mu \mathrm{m}$ de diâmetro e a resina utilizada é à base de fenol-formaldeído e breu, produzido da resina secretada por certas árvores. Em 1975, surgiram os filtros feitos com fibras poliméricas, tipo eletreto, largamente utilizados até os dias de hoje (FELTHAN, 1976; TORLONI; VIEIRA, 2003).

Eletreto é um material dielétrico que absorve ou retém cargas elétricas de forma duradoura, passando a ter propriedades magnéticas (polarização).

Vários autores indicam que os principais métodos para obtenção do carregamento da fibra são por efeito corona, triboelétrico e por indução (BROWN, 1993; KRAVTSOV et al., 2000; TORLONI, 2002).

\section{Não tecidos}

Os não tecidos, conhecidos também como tecidos não tecidos (TNT), são formados por fibras sintéticas. O uso do TNT tem se popularizado e sua aplicação pode ser vista praticamente em todo o lugar, inclusive no uso de filtros e respiradores. Entre um grande número de fibras disponíveis no mercado, os materiais mais utilizados na fabricação de não tecido são: poliolefinas, poliéster e rayon (DRUCKETT, 1999).

Devido às aplicações cada vez mais técnicas dos não tecidos, os requisitos com relação às propriedades das fibras acabaram tornando-se muito importantes. O polipropileno (PP) e o polietileno (PE) são os dois membros mais comuns da família das olefinas, sendo que o PP em comparação com o PE é muito mais versátil, no sentido de tornar-se fibras, e tem sido usado com muito sucesso em vários produtos e mercados em todo o mundo. A fibra de PP funde-se entre $160^{\circ} \mathrm{C}$ e $170^{\circ} \mathrm{C}$, caracterizando uma boa vantagem, pois possibilita uma grande margem de ajustes de temperaturas durante o processo de fabricação de filtros ou PFF, permitindo que elas se mantenham macias, mesmo após o processamento a quente (HEGDE; DAHIYA; 
KAMATH, 2004). O processamento a quente, em geral, é utilizado para unir as bordas dos filtros ou para conformá-los ${ }^{4}$.

Os métodos de processamento das poliolefinas mais utilizados para a fabricação de filtros para particulados, usados em EPR, são Melt Blown (MB), Spunbonds (SB) e Electrospining (ES) (HEGDE; DAHIYA; KAMATH, 2004).

As fibras produzidas pelo processo Spunbonded possuem os diâmetros entre $10 \mu \mathrm{m}$ e $35 \mu \mathrm{m}$. As produzidas em Meltblown são descontínuas e muito finas, com diâmetros menores que 10 micra. As nanofibras, em geral, possuem o diâmetro menor que um mícron (20 nanômetros a 200 nanômetros). As nanofibras são fabricadas pelo processo eletrospining e, devido ao diâmetro diminuto destas fibras, é muito comum o seu uso em filtros e respiradores de alta eficiência. A trama gerada pelas nanofibras favorece os mecanismos mecânicos de captura das partículas em suspensão.

\section{Filtração}

Segundo Torloni e Vieira (2003), existe quase uma dezena de mecanismos que atua simultaneamente na captura de partículas quando estas passam pelos vãos entre as fibras de uma camada do filtro.

Os filtros utilizados em proteção respiratória, quando em uso, obrigam, por efeito da pressão negativa gerada pela respiração ou por ventilação forçada, que ocorra um arraste do ar contendo os contaminantes do ambiente, sendo estes forçados a passar pelo filtro. As partículas deslocam-se por uma trajetória extensa, que é afetada pelo emaranhado das fibras. Neste longo trajeto, elas acabam chocando-se com a parede das mesmas. A retenção das partículas na superfície das fibras é provocada pelas forças de Van der Waals (TORLONI; VIEIRA, 2003).

Filtros eletretos são usados largamente em aplicações que requerem alta eficiência de filtração e baixa perda de carga. Estes filtros utilizam-se dos mecanismos eletrostáticos de captura de partículas em adição aos mecanismos mecânicos convencionais (ROMAY et al., 1998).

A eficiência de captura por interceptação de partículas micrométricas de uma única fibra, com diâmetro aproximado de $20 \mu \mathrm{m}$ (diâmetro típico de fibras animais, vegetais ou sintéticas), fabricada por processos de tecelagem, é extremamente baixa. Por exemplo: partículas de diâmetros entre $2 \mu \mathrm{m}$ e $3 \mu \mathrm{m}$ possuem uma penetração entre $47 \%$ e $22 \%$. Esta mesma fibra, quando carregada eletrostaticamente, passa a ter uma penetração de $0,06 \%$ a $0,03 \%$, sendo os valores de penetração aproximados (BROWN, 1993).
As fibras carregadas eletrostaticamente atraem tanto partículas carregadas quanto neutras. A captura das partículas com cargas negativas ocorre por meio da atração por forças Coulombianas. A captura de partículas neutras ocorre pela ação das forças de polarização.

Segundo Lowkis e Motyl (2001), as partículas carregadas têm a trajetória desviada quando passam pelo campo elétrico gerado pelas fibras elétricas. Já as partículas neutras são polarizadas e movem-se no sentido do campo elétrico.

Filtros mecânicos são menos efetivos quanto à filtração de partículas menores que $1 \mu \mathrm{m}$, isto porque as fibras, tanto naturais, como sintéticas, possuem um diâmetro da ordem de $20 \mu \mathrm{m}$ (DROUIN, 2000). Ainda que o uso de micro fibras ou fibra de vidro venha a aumentar a eficiência da filtração mecânica, o que acarretará um grande aumento na resistência à respiração.

Os principais mecanismos de captura das partículas por meios mecânicos são: interceptação direta, impactação inercial, deposição por difusão e deposição gravitacional.

\section{Pré-condicionamento dos filtros}

Buscando reproduzir as possíveis condições de trabalho, processamento e/ou armazenamento em alta temperatura a que os filtros ou PFF possam estar expostos, as normas americanas, européias e brasileiras de aprovação para filtros ou PFF para partículasdefinem condições de pré-condicionamento dos filtros antes de testá-los contra a penetração inicial. O Quadro 2 fornece uma comparação destas normas e respectivas condições de pré-condicionamento.

\section{Resultados e discussão}

Após a seleção e a identificação das amostras, iniciaram-se os testes, conforme planejamento pré-estabelecido. Como parte deste planejamento, objetivando-se determinar as faixas de temperatura a serem utilizadas no estudo e inclusive a decisão da aplicação ou não da umidade no experimento, foi conduzido um estudo preliminar em que as amostras foram submetidas a altas temperaturas por um dado espaço de tempo e com alta umidade. A umidade foi criada mediante colocação de beckers com água destilada no interior da estufa e a medição da umidade foi feita por meio de plotagem dos resultados das temperaturas de termômetros de bulbo seco e bulbo úmido, em carta psicométrica. Por meio de teste da penetração inicial, antes

\footnotetext{
${ }^{4}$ Conforme informações verbais recebidas de fabricantes de mantas filtrantes, alguns tipos de filtros, dependendo da forma geométrica e/ou tipo de processo usado para a soldagem das suas bordas (geralmente ultrassom ou a quente), podem ficar expostos a temperaturas na faixa de $90^{\circ} \mathrm{C}$ a $140^{\circ} \mathrm{C}$, por períodos de até 15 segundos.
} 
Quadro 2 Comparação entre normas e requisitos de pré-condicionamento com relação à temperatura e à umidade relativa (UR)

\begin{tabular}{|c|c|c|c|c|c|}
\hline Classe Filtro & EN $143^{(1)}$ & $E N 149^{(2)}$ & 42 CFR $84^{(3)}$ & NBR $13697^{(4)}$ & NBR $13698^{(5)}$ \\
\hline $\begin{array}{l}\text { P1 } \\
\text { P2 } \\
\text { P3 }\end{array}$ & $\begin{array}{c}24 \mathrm{~h} \times 70^{\circ} \mathrm{C} \\
24 \mathrm{~h} \times-30^{\circ} \mathrm{C} \\
\text { UR seco }\end{array}$ & & & $\begin{array}{c}24 \mathrm{~h} \times 65^{\circ} \mathrm{C} \\
(\mathrm{UR} \mathrm{seco}) \\
24 \mathrm{~h} \times-15^{\circ} \mathrm{C}\end{array}$ & \\
\hline $\begin{array}{l}\text { N95 } \\
\text { P100 }\end{array}$ & & & $\begin{array}{c}25 \mathrm{~h} \times 38^{\circ} \mathrm{C} \\
\text { UR } 85 \%\end{array}$ & & \\
\hline $\begin{array}{c}\text { PFF1 PFF2 } \\
\text { PFF3 }\end{array}$ & & $\begin{array}{c}24 \mathrm{~h} \times 70^{\circ} \mathrm{C} \\
24 \mathrm{~h} \times-30^{\circ} \mathrm{C} \\
\text { UR seco }\end{array}$ & & & $\begin{array}{c}24 \mathrm{~h} \times 65^{\circ} \mathrm{C} \\
(\mathrm{UR} \mathrm{seco}) \\
24 \mathrm{~h} \times-15^{\circ} \mathrm{C}\end{array}$ \\
\hline
\end{tabular}

(1) (COMISSÃO EUROPÉIA DE NORMALIZACC̃̃O, 2000)

(2) (COMISSÃO EUROPÉIA DE NORMALIZAÇÃO, 2001)

(3) (ESTADOS UNIDOS, 1995)

(4) (ASSOCIAÇÃO BRASILEIRA DE NORMAS TÉCNICAS, 1996a)

(5) (ASSOCIAÇÃO BRASILEIRA DE NORMAS TÉCNICAS, 1996b)

de submeter as amostras ao condicionamento específico e a cada período de exposição, constatou-se a degradação da carga eletrostática mediante a exposição à temperatura e/ou à umidade.

Foram testadas amostras de 7 fabricantes distintos, variando a temperatura de $80^{\circ} \mathrm{C}$ a $126^{\circ} \mathrm{C}$ com umidades entre $60 \%$ a $85 \%$ e tempo de permanência entre 1 e 94 horas na respectiva temperatura. Neste estudo preliminar, não houve preocupação com a classificação ou o tipo de filtro, mas sim com a variação dos resultados de penetração após cada condição de temperatura/umidade e/ou fabricante.

A amostra mantida a $80^{\circ} \mathrm{C}$ e $85 \%$ de umidade foi testada a intervalos de 24 horas, com penetração inicial, antes do condicionamento, de $0,35 \%$, sendo os resultados subsequentes de 1,7\%, 2,16\%, 2,68\% e 2,77\%. As amostras mantidas a $110^{\circ} \mathrm{C}$ por uma hora tiveram incrementos na penetração inicial da ordem de $0,4 \%$ a $6,0 \%$, dependendo do fabricante. As amostras mantidas nas temperaturas de $113^{\circ} \mathrm{C}$ a $126^{\circ} \mathrm{C}$ por uma hora variaram de $-0,11 \%$ a $2,5 \%$. Finalmente, as amostras mantidas a $110^{\circ} \mathrm{C}$ durante 12 horas tiveram uma variação de $3,4 \%$ a $10 \%$.

Devido à grande dificuldade em manter a umidade e fazer uma medição confiável desta dentro da estufa, decidiu-se por não considerar esta variável no estudo final, ficando apenas as variáveis temperatura versus tempo.

Em função da obtenção de um resultado negativo da penetração dentre as diferenças dos resultados inicial e final nos testes preliminares, considerou-se a possibilidade da razão deste desvio de medição ser inerente ao próprio método e/ou equipamento de medição. Para sanar esta dúvida, decidiu-se por realizar uma série de testes de penetração inicial em uma mesma amostra como segue.

Após testar todas as amostras coletadas quanto à penetração inicial, foram escolhidos dois filtros, sendo um classe PFF1 com o maior valor de penetração e outro classe PFF2 com a menor penetração; desta forma, foi possível verificar o comportamento de dois filtros que apresentaram os resultados mais extremos da população. Os dois filtros selecionados foram então submetidos a uma sequência de 10 testes consecutivos, repetindo-se inclusive a montagem do filtro no dispositivo de teste e deste no suporte do equipamento. Os resultados dos 10 testes consecutivos referentes à penetração inicial e à resistência à respiração foram compilados e podem ser observados nos Gráficos 1 e 2 .

O comportamento quanto à penetração inicial e à resistência à respiração em ambas as amostras mostrou-se muito semelhante, ou seja, a penetração teve uma diminuição e a resistência à respiração aumentou a cada sequência de testes. Este comportamento ocorre devido à constante deposição de partículas de $\mathrm{NaCl}$ sobre a superfície das fibras do filtro causando um certo "entupimento", retendo, assim, mais e mais partículas, diminuindo a penetração e aumentando a resistência, ou seja, as partículas sólidas se amontoam sobre as fibras, contribuindo, desta forma, para a melhora dos mecanismos de filtração mecânica, aumentando a perda de carga.

Este tipo de comportamento já era esperado, como demonstram os estudos de Chih-Chieh, Lehtimäki e Willeke (1993), Barrett e Rousseau (1998) e Moyer e Bergman (2000), comprovando, assim, a eficácia do equipamento e dos dispositivos empregados na pesquisa.

Devido à grande variação de resultados entre as amostras de fabricantes diferentes, decidiu-se coletar uma quantidade maior de amostras, com maior variação dos fabricantes de filtros. Um total de 32 amostras foi submetido ao condicionamento e a teste de penetração. O Quadro 1 detalha os tipos de amostras.

Com base nos resultados obtidos por outros pesquisadores, em exigências normativas e na observação 
Penetração X Resistência - PFF1

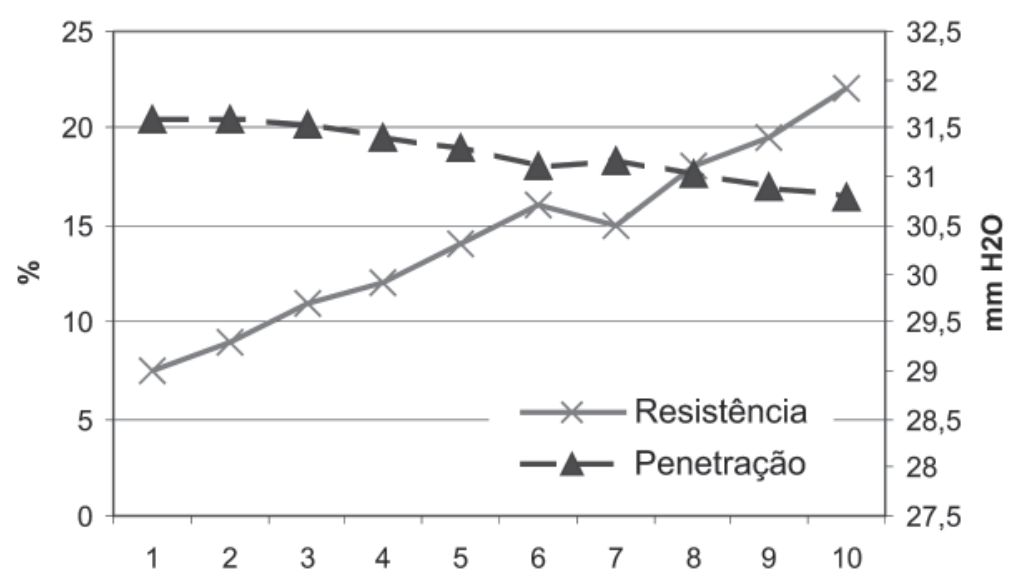

Gráfico 1 Repetição de 10 testes seqüenciais, com peça semifacial filtrante 1 - PFF1, de penetração inicial e resistência à respiração

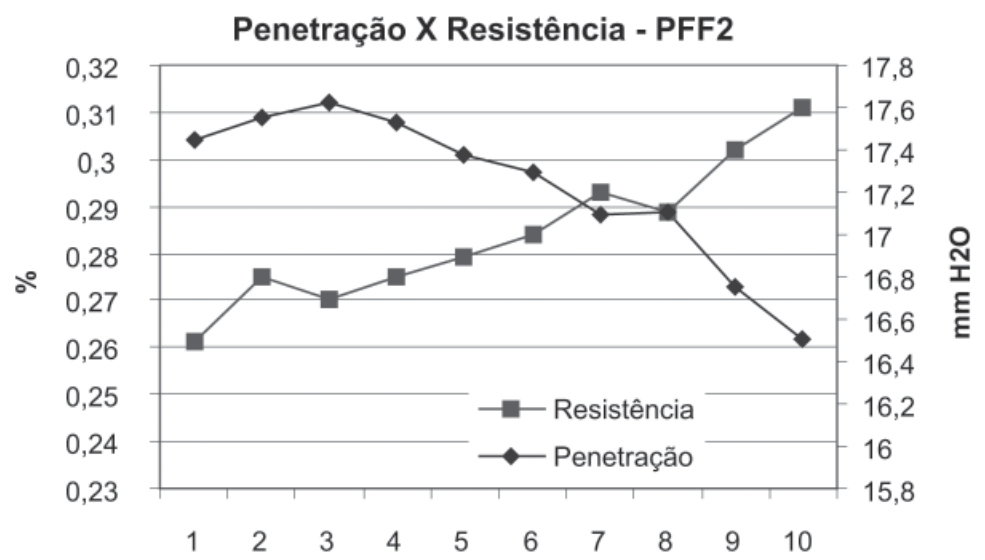

Gráfico 2 Repetição de 10 testes sequienciais, com peça semifacial filtrante 2 - PFF2, de penetração inicial e resistência à respiração

dos resultados dos testes preliminares, decidiu-se por adotar os seguintes parâmetros e conduta na continuidade dos testes.

A temperatura inicial ficou definida em $75^{\circ} \mathrm{C}$ com intervalo de 24 horas entre as medições. O período total de testes teve a duração de 264 horas.

As amostras de controle foram escolhidas após serem testadas todas as 32 amostras previamente selecionadas. Após os testes iniciais, foram selecionadas 6 amostras, sendo 3 de cada fabricante, que apresentaram os menores e os maiores resultados de penetração inicial, das quais, duas de cada grupo foram mergulhadas por $10 \mathrm{mi}-$ nutos em isopropanol (álcool isopropílico), sendo secas ao ar livre em temperatura ambiente, e a terceira amostra de cada grupo foi mantida como controle. Ou seja, dois filtros foram separados para servirem de controle.
Todas as amostras foram medidas quanto à penetração e à resistência à respiração antes e depois do procedimento. As amostras de controle passaram a ser testadas nos mesmos intervalos das outras amostras, porém não foram expostas à temperatura.

O isopropanol foi utilizado a fim de verificar a influência da carga eletrostática nas amostras coletadas, pois o uso do mesmo resulta na remoção da carga eletrostática das fibras dos filtros. Este procedimento foi descrito em outros estudos, em que foi demonstrada esta capacidade singular atribuída ao isopropanol (CHIH-CHIEH; SHENG-HSIU, 1998; MARTIN JUNIOR; MOYER, 2000). A penetração foi medida antes e depois da remoção da carga eletrostática por tratamento com isopropanol. Após estas medições, as 4 amostras foram expostas à temperatura de $75^{\circ} \mathrm{C}, 90^{\circ} \mathrm{C}$ e $110^{\circ} \mathrm{C}$ e passaram a ser monitoradas a cada 24 horas, num total de 144 horas (Gráfico 3), obje- 
tivando-se, assim, observar a variação da penetração com um filtro supostamente isento de carga eletrostática.

Observou-se que o filtro classe P2 ou N95 (amostras 1 e 2 do fabricante 8) apresentou maior dificuldade na remoção total das cargas eletrostáticas pelo uso do isopropanol, pois se constatou que, ao longo do tempo, o filtro mantido à temperatura de $75^{\circ} \mathrm{C}$ durante 24 horas apresentou um aumento na penetração média de $21 \%$ para $23 \%$, variando mais $13 \%$ à temperatura de $90^{\circ} \mathrm{C}$. Os resultados destes testes podem ser observados no Gráfico 3.

As 26 amostras remanescentes também foram expostas às temperaturas de $75^{\circ} \mathrm{C}, 90^{\circ} \mathrm{C}$ e $110^{\circ} \mathrm{C}$ e passaram a ser monitoradas a cada 24 horas, sendo, porém, o período total do teste de 264 horas, ou seja, 11 dias (Gráfico 4). Exceto para as amostras de controle, o procedimento também seguiu o estabelecido, testando as amostras antes e após condicionamento térmico.

Tratamento com Isopropanol

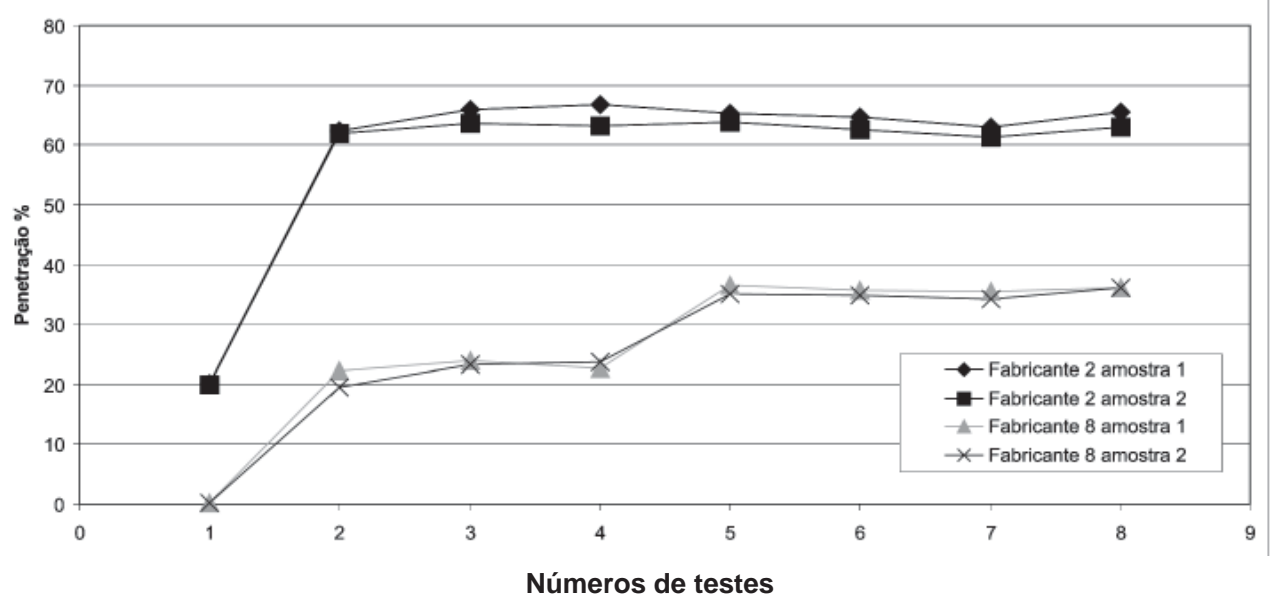

Teste número 1 - amostras testadas como recebido em temperatura ambiente

Teste número 2 - amostras testadas após tratamento com isopropanol

Testes 3 e 4 - amostras testadas após condicionamento térmico à $75^{\circ} \mathrm{C}$ em intervalos de $24 \mathrm{~h}$

Testes 5 a 7 - amostras testadas após condicionamento térmico à $90^{\circ} \mathrm{C}$ em intervalos de $24 \mathrm{~h}$

Teste 8 - amostra testada após condicionamento térmico à $110^{\circ} \mathrm{C}$ em intervalo de $24 \mathrm{~h}$

Gráfico 3 Aumento da penetração inicial em filtros para partículas, testados com $\mathrm{NaCl}$, em função da remoção das cargas elétricas por efeito do Isopropanol e posterior exposição à temperaturas de $75^{\circ} \mathrm{C}$ a $110^{\circ} \mathrm{C}$

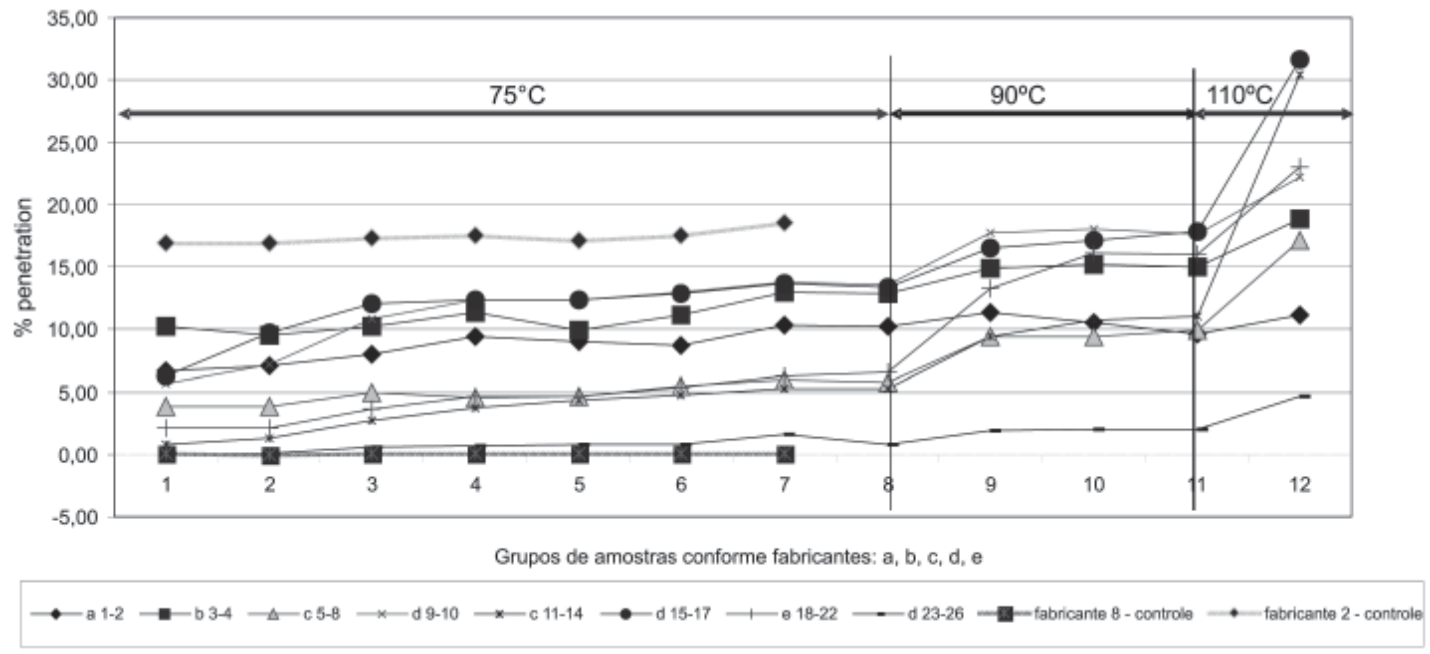

Gráfico 4 Valores médios de penetração de cada um dos grupos de amostras de filtros de diferentes fabricantes (a, b, c, d, e) e das amostras de controle, em função do tempo de exportação e incremento da temperatura 
Os resultados dos testes de penetração, antes e após o condicionamento térmico nos dados intervalos de tempo, apresentaram uma variação máxima para a mesma amostra de $2,53 \%$ até $18 \%$. Dependendo do fabricante, amostras mantidas a $75^{\circ} \mathrm{C}$ no intervalo de 24 a 168 horas variaram a penetração de $9,26 \%$ a $12,55 \%$. Para incremento da temperatura até $90^{\circ} \mathrm{C}$, houve variação da ordem $1,7 \%$ a $18 \%$, já o aumento da temperatura para a faixa de $110^{\circ} \mathrm{C}$ por 24 horas causou danos estruturais em alguns filtros, não sendo possível prosseguir com os estudos, porém os que não foram danificados tiveram um aumento da penetração em até $18 \%$.

\section{Conclusões}

Semelhantemente aos resultados encontrados por outros pesquisadores, constatou-se uma forte influência da temperatura em função do tempo na eficiência dos filtros para particulados.

Ao comparar as variações de penetração encontradas com os requisitos normativos, observou-se que $60 \%$ dos filtros originalmente classificados como P1 ou N95, após 120 horas de exposição a $75^{\circ} \mathrm{C}$, apresentaram níveis de penetração superiores à faixa de aprovação definidas nas normas descritas neste trabalho.

A norma 42 CFR 84 (ESTADOS UNIDOS, 1995) determina pré-condicionamento entre $65^{\circ} \mathrm{C}$ e $70^{\circ} \mathrm{C}$ por períodos de 24 horas. Neste caso, nenhum dos filtros testados seria reprovado, pois o tempo de exposição seria ainda muito pequeno e a temperatura ainda estaria $5^{\circ} \mathrm{C}$ menor que a verificada nos testes descritos nesta pesquisa.

Considerando as condições térmicas no ambiente de trabalho, é justo questionar se os filtros testados continuariam a oferecer, ao longo do tempo, a proteção adequada ao usuário, visto que os filtros podem ficar expostos por períodos maiores que 24 horas a temperaturas superiores a $70^{\circ} \mathrm{C}$.

\section{Referências}

ASSOCIAÇÃO BRASILEIRA DE NORMAS TÉCNICAS. NBR 12543: equipamentos de proteção respiratória: terminologia. Rio de Janeiro, 1999.

. NBR 13697: equipamentos de proteção respiratória: filtros mecânicos. Rio de Janeiro, 1996a.

. NBR 13698: equipamentos de proteção respiratória: peça semifacial filtrante para partículas. Rio de Janeiro, 1996b.

BARRETT, L. W.; ROUSSEAU, A. D. Aerosol loading performance of electret filter media. American Industrial Hygiene Association Journal, United States, v. 59, n. 8, p. 532-539, Aug. 1998.
Observou-se, durante o estudo, que a amostra de um dos fabricantes permaneceu praticamente inalterada ao longo do tempo, comprovando a existência da possibilidade da não ocorrência da degradação em determinados elementos filtrantes em função da temperatura e do tempo de exposição.

Os resultados dos testes realizados durante a pesquisa levam à conclusão de que temperaturas de $75^{\circ} \mathrm{C}$ podem causar variações significativas na eficiência de filtração dos filtros, dependendo do fabricante. Para a temperatura de $90^{\circ} \mathrm{C}$, a penetração aumentou ainda mais, praticamente a mesma variação obtida com exposição a $75^{\circ} \mathrm{C}$ em determinado período, no entanto 2,3 vezes menor. O uso de temperatura muito elevada, ou seja, $110^{\circ} \mathrm{C}$, danifica os filtros, todavia, nos filtros não danificados, ocorreu um aumento ainda maior da penetração do que o observado nas temperaturas de $75^{\circ} \mathrm{C}$ e $90^{\circ} \mathrm{C}$ em um tempo ainda menor. Desta forma, quanto maior a temperatura, menor será o tempo de exposição para se obter os mesmos valores de aumento da penetração.

Finalmente, amostras do mesmo fabricante, porém de lotes diferentes, apresentaram diferenças consideráveis nos resultados de penetração quando acondicionadas nas mesmas condições de temperatura e por um mesmo período. Consequentemente, conclui-se que existem variáveis nos processos de fabricação das mantas e/ou dos filtros que influenciam diretamente na sensibilidade dos filtros com relação à exposição a elevadas temperaturas. Conforme resultados obtidos, percebe-se que não existe uma repetibilidade dos processos de fabricação tanto do material filtrante, como do próprio filtro. Sendo assim, são necessárias ações por parte dos fabricantes, principalmente dos fabricantes do material filtrante, no sentido da definição das variáveis de processo para uma posterior validação dos efeitos destas. Desse modo, poderá haver um aumento da confiabilidade, resultando na repetibilidade dos resultados de penetração inicial nos lotes dos materiais filtrantes e dos filtros quando expostos a altas temperaturas e por longos períodos de tempo.

BROWN, R. C. Review: modern concepts of air filtration applied to dust respirators. The Annals of Occupational Hygiene, England, v. 33, n. 4, p. 615-644, Jan. 1989.

Air filtration: an integrated approach to the theory and applications of fibrous filters. Oxford: Pergamon Press, 1993.

. Filtration in industrial hygiene. American Industrial Hygiene Association Journal, United States, v. 62, n. 5, p. 633-643, Sept. 2001.

CAMARGO, O. F. Estudo de desempenho de filtros para particulados e seleção de respiradores para uso em mineradoras. 2007. 120 f. Dissertação (Mestrado)- 
Escola Politécnica, Universidade de São Paulo, São Paulo, 2007.

CHENG, Y. S.; HOLMES, T.; FAN, B. Evaluation of respirator filters for asbestos fibers. Journal of Occupational and Environmental Hygiene, United States, v. 3, n. 1, p. 26-35, Jan. 2006.

CHIH-CHIEH, C.; LEHTIMÄKI, M.; WILLEKE, K. Loading and filtration characteristics of filtering facepieces. American Industrial Hygiene Association Journal, United States, v. 54, n. 2, p. 51-60, Feb. 1993.

CHIH-CHIEH, C.; SHENG-HSIU, H. The effects of particle charge on the performance of a filtering facepiece. American Industrial Hygiene Association Journal, United States, v. 59, n. 4, p. 227-233, Apr. 1998.

COMISSÃO EUROPÉIA DE NORMALIZAÇÃO. EN 143: respiratory protective devices: particle filters: requirements, testing, marking. England, 2000.

. EN 149: respiratory protective devices: filtering half masks to protect against particles: requirements, testing, marking. England, 2001.

DROUIN, B. Triboelectric blend enhance air filtration. Filtration \& Separation, v. 37, n. 9, p. 20-23, Nov. 2000.

DRUCKETT, K. Materials science \& engineering 554: nonwovens science and technology II. 1999. Disponível em: <http://www.engr.utk.edu/mse/Textiles/index. html>. Acesso em: 1 jul. 2007.

ESTADOS UNIDOS. Code of Federal Regulations. Department of Health and Human Services. Public Health Services. 42 CFR 84: subpart K: non powered air purifying particulate respirator. Rockville, 1995.

FELTHAN, F. J. Second thoughts on the hansen electrostatic filter. Filtration $\&$ Separation, v. 13, p. 467-469, Sept./Oct. 1976.

FJELD, R. A.; OWENS, T. M. The effect of particle charge on penetration in an electret filter. IEEE Transactions on Industry Applications, United States, v. 24, n. 4, p. 725-731, July/Aug. 1988.

HEGDE, R. R.; DAHIYA, A.; KAMATH, M. G. Success story: polypropylene in the textile industry. 2004
Disponível em: <http://www.engr.utk.edu/mse/textiles/ olefin\%20fibers.htm>. Acesso em: 1 jul. 2007.

KRAVTSOV, A. et al. The electret effect in polypropylene fibers treated in a corona discharge. Advances in Polymer Technology, United States, v. 19, n. 4, p. 312-316, 2000.

LOWKIS, B.; MOTYL, E. Electret properties of polypropylene fabrics. Journal of Electrostatics, Netherlands, v. 51-52, p. 232-238, May 2001.

MARTIN JUNIOR, S. B.; MOYER, E. S. Electrostatic respirator filter media: filter efficiency and most penetrating particle size effects. Applied Occupational and Environmental Hygiene, England, v. 15, n. 8, p. 609-617, Aug. 2000.

MOTYL, E.; LOWKIS, B. Effect of air humidity on charge and lifetime of PP electret nonwovens. Fibers $\&$ Textil in Eastern Europe, Poland, v. 14, n. 5, p. 39-42, Jan./Dec. 2006.

MOYER, E. S.; BERGMAN, M. S. Electrostatic N-95 respirator filter media efficiency degradation resulting from intermittent sodium chloride aerosol exposure. Applied Occupational and Environmental Hygiene, England, v. 15, n. 8, p. 600-608, Aug. 2000.

ROMAY, F. J. et al. Experimental study of electrostatic capture mechanisms in commercial electret filters. Aerosol Science and Technology, United States, v. 28, n. 3, p. 224-234, Jan. 1998

TORLONI, M. (Coord.). Programa de proteção respiratória: recomendações seleção e uso de respiradores. 3. ed. São Paulo: Fundacentro, 2002.

TORLONI, M.; VIEIRA, A. V. Manual de proteção respiratória. São Paulo: ABHO, 2003.

TSI INCORPORATED. TSI model 8110: a salt aerosol generator. Shoreview: TSI, 2004. (Instruction Manual). Automated filter tester operation and service manual P/N 1980207. Certitest ${ }^{\circledR}$ model 8127/8130. Shoreview: TSI, 2008. 